\title{
DEMONSTRATION OF A GAIN FLATTENED OPTICAL AMPLIFIER WITH A MICROMECHANICAL EQUALIZER ELEMENT
}

\author{
J.A. Walker, J.E. Ford, and K.W. Goossen \\ Lucent Technologies, Bell Laboratories \\ Holmdel, NJ 07733
}

\begin{abstract}
Free-space optics and micromechanics are combined to produce a wavelength spectrum equalizer for use in dense WDM telecommunications networks. Equalization of the gain spectrum of an erbium doped fiber amplifier is demonstrated wherein the variation of gain over $23 \mathrm{~nm}$ is flattened from $>13 \mathrm{~dB}$ to $\pm 0.25 \mathrm{~dB}$.

\section{INTRODUCTION}

In order to accommodate the rapid growth in traffic due to the Internet and data transmission, telecommunications providers are moving rapidly toward the use of dense wavelength division multiplexing (DWDM) solutions. A vital component in DWDM systems is the Erbium-Doped Fiber Amplifier (EDFA). In practice, the usable spectral range of EDFAs has been limited to roughly $12 \mathrm{~nm}$ due to the non-uniform gain profiles of these devices. In a chain of amplifiers, gain non-uniformity of one amplifier is further increased in the next stage, leading to transmission errors. A limitation in spectral width translates to a limitation on number of wavelength channels and therefore higher system costs. Fixed filters, such as long period fiber gratings, have been used to expand the usable spectrum to roughly $40 \mathrm{~nm}$, but these cannot respond to changes occurring over the lifetime of the EDFA. Over the lifetime of a system, the power from the laser used to pump the EDFA will change, resulting in a change of the EDFA gain profile. Presently, operators choose sub-optimal performance at system inception to allow for changing pump conditions and an increase in system lifetime. Additionally, as DWDM systems become more complex network reconfiguration, such as wavelength add/drop, is becoming highly desirable. Reconfiguration leads to changing input power distribution to the EDFAs again resulting in transmission errors [1]. We present here a WDM equalizer with the ability to flatten the output of an EDFA with the capability to adapt to changes occurring over the lifetime of the amplifier.
\end{abstract}

\section{EQUALIZER DEVICE TECHNOLOGY}

A WDM equalizer system was reported earlier [2] that utilized segmented MARS elements that allowed independent control over each wavelength channel. While this type of device is very useful for equalizing the intensities of a set of given wavelength channels, the channel spacing is fixed by the spatial resolution of the elements and the optics in the system. In the work we report here, we demonstrate passband-free equalization that allows for equalization independent of channel spacing, as well as continuous smooth shaping of the wavelength spectrum.

In both equalizer configurations, we use the same device technology as that of the MARS optical modulator [3], which is based on optical interference effects between a dielectric film suspended above a substrate and the substrate. For a single element MARS device operating at a wavelength of $1.54 \mu \mathrm{m}$, a $\lambda$ 4-thick membrane (1950 $\AA$ ) of silicon nitride is formed suspended above a silicon substrate with an air gap equal to $3 \lambda / 4$ $(1.155 \mu \mathrm{m})$. The air gap and suspended nitride film form a multilayer dielectric mirror stack or low finesse Fabrey-Perot cavity. If a voltage is applied between electrodes formed on top of the nitride film and the substrate, the membrane is moved toward the substrate thereby changing the reflectivity of the mirror stack. Attenuation levels of up to $30 \mathrm{~dB}$ over a broad wavelength range are attainable as shown in Figure 1.

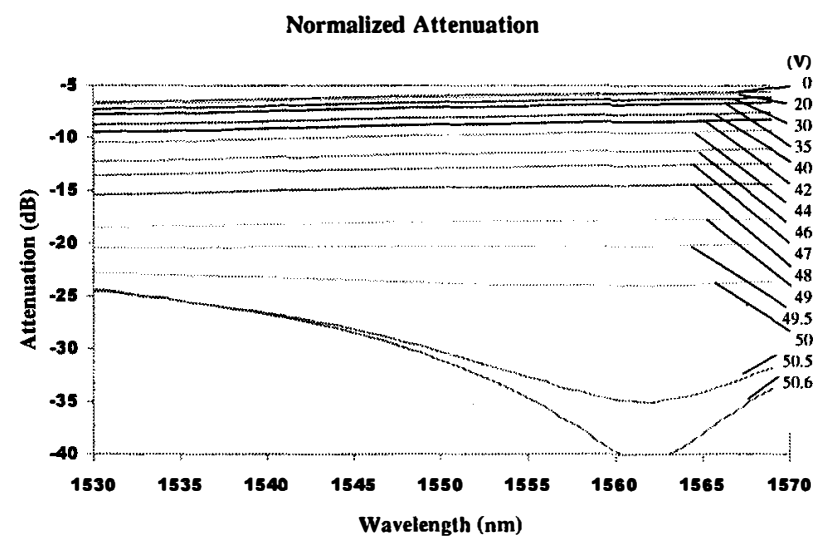

Figure 1. Measured attenuation spectra of a MARS attenuator vs. applied voltage.

In the case of a WDM equalizer, we form an optically active region of $35 \mu \mathrm{m}$ by roughly $1 \mathrm{~mm}$ in length. 34 electrically independent electrode pairs are arrayed along the length of the device on a $28-\mu \mathrm{m}$ pitch as shown in Figure 2. Figure 3 shows the free-space optical system, which directs the output of an EDFA with a spatial spread of wavelength along the 1-mm long length of the equalizer device. In this system, the input signal comes in via Port 1 and is collimated by lens system 1 . The collimated beam is then spread out spatially by a diffraction grating and focussed onto the equalizer optical window by a focussing lens. Control voltages applied to the independent electrodes create a smoothly varying deflection of the nitride membrane. This deflection shape results in a reflectivity profile across the length of the optical window yielding the desired flattened spectrum. The shaped spectrum is reflected back through the focus lens, reflected back off the grating and is finally sent to Port 2 (output) via a fold mirror. 


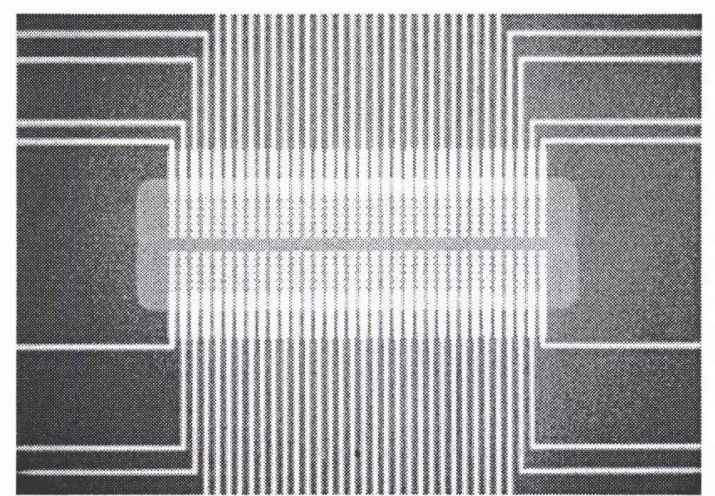

Figure 2. A WDM equalizer element with an optically active region of $35 \mu \mathrm{m}$ by $950 \mu \mathrm{m}$.

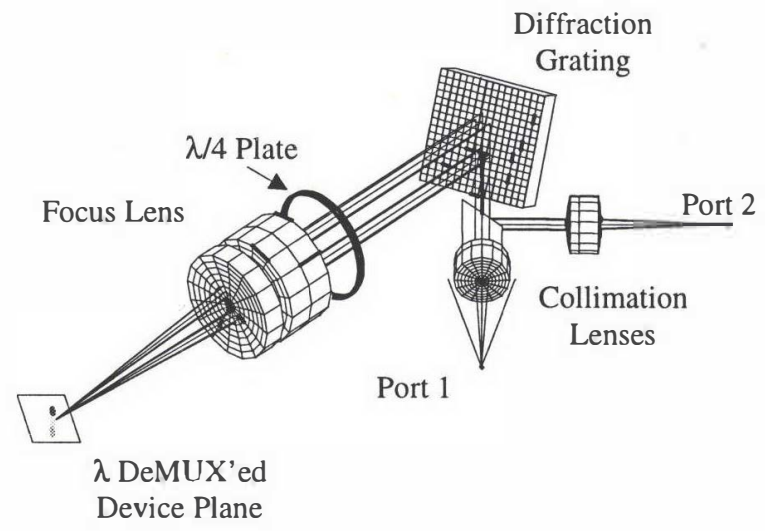

Figure 3. WDM equalizer free-space optics system.

\section{EXPERIMENT AND RESULTS}

The output of an EDFA amplifier with no input signal is its amplified spontaneous emission (ASE). The ASE is representative of the spectral shaping an EDFA would add to a flat input spectrum. Figure 4 shows the ASE output from a typical amplifier, the ASE signal transmitted through the equalizer without control, and the flattened output from the equalizer. As shown in Figure 4, the ASE input exhibits variation in intensity of over $13 \mathrm{~dB}$ across a 23- $\mathrm{dB}$ spectrum. The region where it is flat to within a few $\mathrm{dB}$ is roughly $12 \mathrm{~nm}$. The transmitted spectrum shows an insertion loss of roughly $8 \mathrm{~dB}$ from the equalizer, but we feel some of this was due to optical system misalignment and expect that the insertion loss could be as low as $3 \mathrm{~dB}$. The flattened spectrum shown in Figure 4 demonstrates the capability of reducing the dynamic range of the input signal from $>13 \mathrm{~dB}$ to $\pm 0.23 \mathrm{~dB}$ over a $23.5 \mathrm{~nm}$ bandwidth. In order to obtain the flat gain response, an additional 2-dB loss penalty was incurred (as evidenced over the range of $1337-1340 \mathrm{~nm})$. The penalty resulted from the inability of the continuous membrane to affect a large change in required deflection over such a short distance as required by the large slope of the gain curve in this region.

\section{CONCLUSIONS}

We have demonstrated the ability to flatten the gain curve of an EDFA amplifier to $\pm 0.23 \mathrm{~dB}$ over a $23.5 \mathrm{~nm}$ spectral width. In present DWDM systems, this would translate to an increase in the number of wavelength channels from 8 to 15 , nearly doubling the capacity of the network. In addition to the increase in capacity, this method of spectral control also provides the ability to adapt to changing gain shape over the lifetime of the EDFA amplifier.

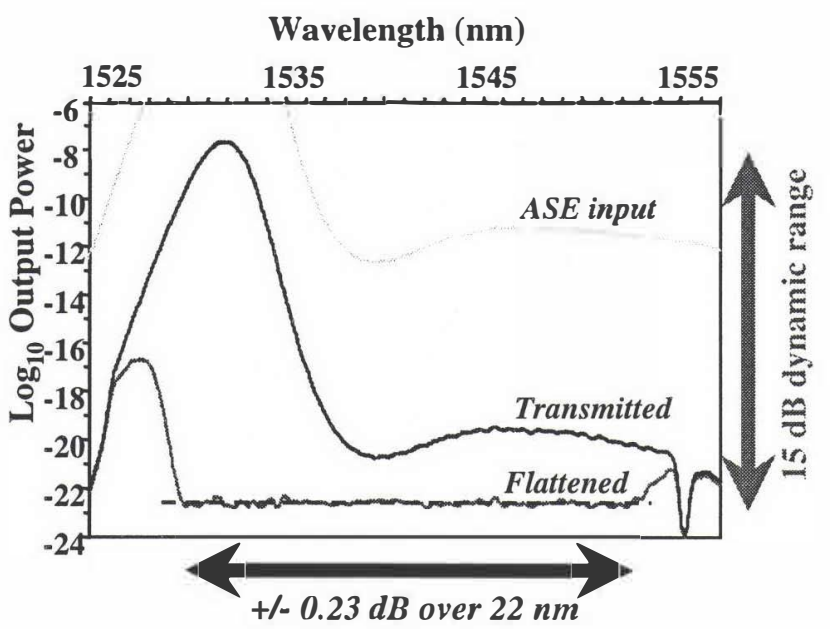

Figure 4. Gain spectra for an erbium-doped fiber amplifier showing the signal directly from the amplifier, through the equalizer without perturbation, and gain flattened by the equalizer.

\section{REFERENCES}

[1] Y. Sun, A.K. Srivastava, J.L. Zyskind, J.W. Sulhoff, C. Wolf, and R.W. Rkach, "Fast Power Transients in WDM Optical Networks with Cascaded EDFAs", Electronics Letters, Vol. 33, pp. 313-314, (1997).

[2] J.E. Ford, J.A. Walker, M. Nuss, and D.A.B. Miller, "32 Channel WDM Graphic Equalizer”, IEEE/LEOS 1996 Summer Topical Meeting on Broadband Optical Networks, Keystone, CO, August 5-7, 1996 pp.26-27.

[3] K.W. Goossen, J.A. Walker, S.C. Arney, "Silicon modulator based on mechanically-active anti-reflection layer with $1 \mathrm{Mbit} / \mathrm{sec}$ capability for fiber-in-the-loop applications", IEEE Photonics Technology Letters, Vol. 6, No. 9, Sept. 1994, pp. 11191121. 